\title{
LETTER
}

\section{Monitoring of neuromuscular blockade: a comparison of train-of-four and the Campbell diagram}

\author{
Elias Baedorf Kassis ${ }^{{ }^{*}} \mathbb{0}$, Sarah Train², Bruce MacNeil ${ }^{3}$, Stephen H. Loring ${ }^{4}$ and Daniel Talmor ${ }^{4}$
}

(C) 2018 Springer-Verlag GmbH Germany, part of Springer Nature and ESICM

Dear Editor,

Neuromuscular blockers (NMB) are frequently used in acute respiratory distress syndrome (ARDS) for severe hypoxemia and ventilator dyssynchrony and have been shown to improve mortality [1]. The benefit may be secondary to removal of spontaneous breathing, dyssynchrony and expiratory efforts [2] thereby decreasing inflammation [3]. NMB dosing protocols, however, do not utilize monitoring of spontaneous breathing activity when determining efficacy [4]. Prior studies used standardized dosing [1] regardless of weight which may underor overdose patients. Additionally current guidelines recommend using train-of-four (TOF) monitoring, a type of peripheral nerve stimulation as a surrogate marker [4], despite the fact that NMB has variable effects on different muscles and the degree of peripheral paralysis may not reflect diaphragmatic and respiratory muscle paralysis [5]. Furthermore, TOF response and interpretation vary as a result of patient factors (edema, temperature, etc.), site of use, technology, and operator experience.

Our group has recently utilized Campbell diagrams (chest wall pressure-volume loops) and esophageal pressure (Pes) to phenotype breathing patterns [2]. In agreement with current hypotheses for NMB benefit, we found that deep sedation and paralysis could restore the passive chest wall and improve end-expiratory lung volumes lost secondary to active expiration. These results also suggested that Campbell diagrams could

\footnotetext{
*Correspondence: enbaedor@bidmc.harvard.edu; ekassis@gmail.com ${ }^{1}$ Division of Pulmonary and Critical Care, Beth Israel Deaconess Medical Center, Harvard Medical School, 330 Brookline Ave, Boston, MA 02215, USA

Full author information is available at the end of the article
}

be utilized to identify passive breathing with real-time monitoring at the bedside. We compared TOF with the Campbell diagram in 13 patients paralyzed with cisatricurium over several measurements while enrolled in an ongoing observational study. Waveforms were collected for 20-40 min per recording and the closest TOF levels obtained from the orbicularis oculi (from 0 to 4 ) were recorded by nursing for routine clinical care. Passive breathing was assessed using the Campbell diagram if breaths showed reproducible upward Pes deflection during inspiration and downward Pes deflection during expiration along the passive chest wall compliance curve. Non-passive breathing was determined if any deviation from this pattern resulted in flow, pressure, or volume changes (to disregard esophageal spasm). STATA (Statacorp LLC) was used for statistics and Windaq (Dataq Instruments) for waveform analysis.

Completely passive breathing was found in 27/34 (79\%) (Fig. 1a) of waveforms while 7/34 (21\%) of waveforms exhibited breathing activity with expiratory efforts accounting for 6 of the 7 (examples in Fig. 1). Patients were often under-paralyzed per TOF score of 4 (11 of 34 ) or over-paralyzed per TOF score of $0-1$ (19/34), with very few patients at goal of $2(4 / 34)$. There appeared to be no difference in NMB dose at different levels of TOF and in active and passively breathing patients, but a larger sample will be needed for generalized estimating equations and linear mixed modeling to account for the lack of independence between observations. Despite these limitations, this analysis suggests that use of TOF may be suboptimal for dosing of NMB and "deeply" paralyzed patients may not have full respiratory muscle paralysis. A targeted approach using the Campbell diagram and esophageal manometry may allow for more

\section{Springer}



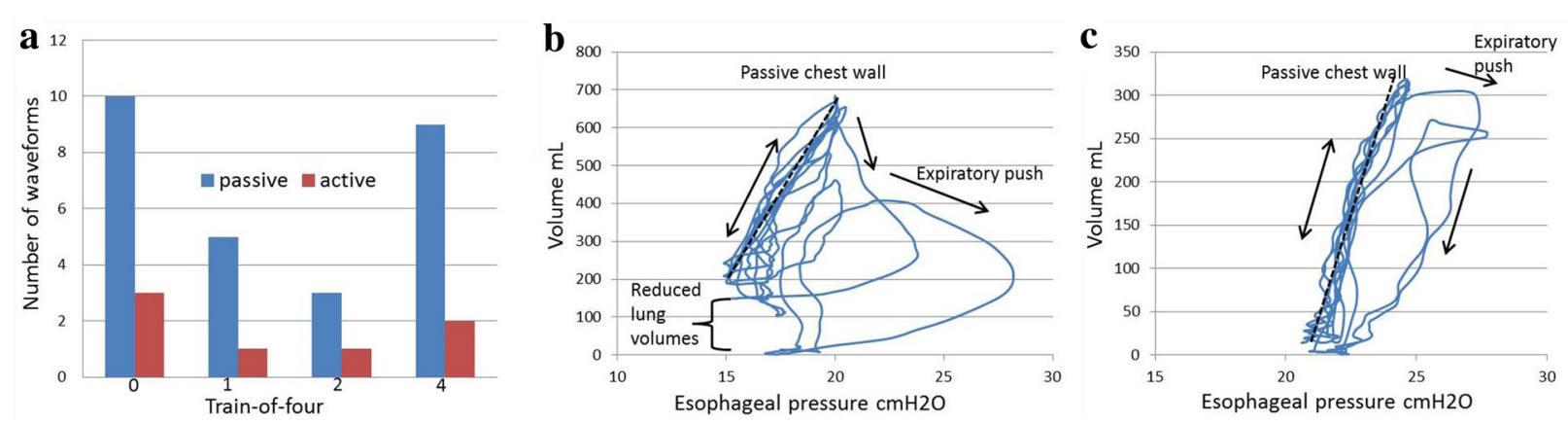

Fig. 1 a Distribution of 34 waveforms from 13 patients with train-of-four (TOF) measurements displaying passive (blue) vs active (red) breathing. Most patients were not kept at goal TOF levels (2/4) and still exhibited active breathing when more "deeply" paralyzed with TOF 0-1. b, c Campbell diagrams (pressure-volume loops of the chest wall) plotting volume change and esophageal pressure (Pes) change (as a surrogate for pleural pressure). Two patients with acute respiratory distress syndrome (ARDS) receiving neuromuscular blockade with cisatricurium exhibiting evidence of spontaneous breathing activity with intermittent expiratory efforts. The passive chest wall is characterized as an upward Pes deviation during the inspiratory phase and downward Pes deviation during the expiratory phase falling along the passive chest wall compliance curve. Active expiratory efforts are characterized by an upward Pes deviation away from the passive chest wall during the expiratory phase. b Patient with expiratory efforts that lower end-expiratory volumes by roughly $200 \mathrm{~mL}$ from below the end-expiratory volume while passively breathing. c More subtle intermittent expiratory efforts with minimal reductions in end-expiratory volume

personalized and optimized care and NMB dosing. We are expanding this analysis to a larger cohort to further this investigation.

\section{Electronic supplementary material}

The online version of this article (https://doi.org/10.1007/s00134-018-5420-5) contains supplementary material, which is available to authorized users.

\section{Author details}

${ }^{1}$ Division of Pulmonary and Critical Care, Beth Israel Deaconess Medical Center, Harvard Medical School, 330 Brookline Ave, Boston, MA 02215, USA.

2 Department of Medicine, Beth Israel Deaconess Medical Center, Harvard Medical School, 330 Brookline Ave, Boston, MA 02215, USA. ${ }^{3}$ Department of Respiratory Care, Beth Israel Deaconess Medical Center, Harvard Medical School, 330 Brookline Ave, Boston, MA 02215, USA. ${ }^{4}$ Department of Anesthesia, Critical Care and Pain Medicine, Beth Israel Deaconess Medical Center, Harvard Medical School, 330 Brookline Ave, Boston, MA 02215, USA.

\section{Funding}

There was no funding for this letter.

\section{Compliance with ethical standards}

\section{Conflicts of interest}

The authors declare no conflicts of interest.

\section{Ethical standards}

All ethical standards were met in writing and submitting this correspondence.
Accepted: 15 October 2018

Published online: 22 October 2018

\section{References}

1. Papazian L, Forel JM, Gacouin A et al (2010) Neuromuscular blockers in early acute respiratory distress syndrome. N Engl J Med 363:1107-1116. https://doi.org/10.1056/nejmoa1005372

2. Baedorf Kassis E, Loring SH, Talmor D (2018) Lung volumes and transpulmonary pressure are decreased with expiratory effort and restored with passive breathing in ARDS: a reapplication of the traditional Campbell diagram. Intensive Care Med 21:21. https://doi.org/10.1007/s0013 4-018-5105-0

3. Sottile PD, Albers D, Moss MM (2018) Neuromuscular blockade is associated with the attenuation of biomarkers of epithelial and endothelial injury in patients with moderate-to-severe acute respiratory distress syndrome. Crit Care 22:63-018-1974-4. https://doi.org/10.1186/s1305 4-018-1974-4

4. Murray MJ, DeBlock H, Erstad B et al (2016) Clinical practice guidelines for sustained neuromuscular blockade in the adult critically ill patient. Crit Care Med 44:2079-2103. https://doi.org/10.1097/ccm.000000000000202 7

5. Moerer O, Baller C, Hinz J, Buscher H, Crozier TA (2002) Neuromuscular effects of rapacuronium on the diaphragm and skeletal muscles in anaesthetized patients using cervical magnetic stimulation for stimulating the phrenic nerves. Eur J Anaesthesiol 19:883-887 